\title{
Rechtsprechung
}

\section{Zur Entfernung einer antisemitischen Schmähplastik an der Stadtkirche zu Wittenberg}

Urteil des OLG Naumburg vom 04.02.2020, Az. 9 U 54/19 (nicht rechtskräftig)

In dem Rechtsstreit hat der 9. Zivilsenat des Oberlandesgerichts Naumburg auf die mündliche Verhandlung vom 21. Januar 2020 durch den Vorsitzenden Richter am Oberlandesgericht Buchloh, die Richterin am Oberlandesgericht Tauscher und den Richter am Oberlandesgericht Dr. Strietzel für Recht erkannt:

Die Berufung des Klägers gegen das am 24.05.2019 verkündete Urteil des Landgerichts Dessau-Roßlau wird zurückgewiesen.

Die Kosten des Berufungsverfahrens werden dem Kläger auferlegt.

Dieses Urteil und das angefochtene Urteil sind ohne Sicherheitsleistung vorläufig vollstreckbar. Der Kläger darf die Vollstreckung der Beklagten durch Sicherheitsleistung in Höhe von $110 \%$ des aus dem Urteil vollstreckbaren Betrages abwenden, wenn nicht die Beklagte vor der Vollstreckung Sicherheit in Höhe des jeweils zu vollstreckenden Betrages leistet.

Die Revision wird zugelassen.

Zudem hat der Senat beschlossen:

Der Streitwert für das Berufungsverfahren wird auf $10.000,00 €$ festgesetzt.

\section{Gründe}

I.

Der Kläger ist Jude und Mitglied einer jüdischen Gemeinde. Die Beklagte ist Eigentümerin der Stadtkirche in ... Auf dem südöstlichen Flügel der Stadtkirche

befindet sich in mehreren Metern Höhe ein Sandsteinrelief, das mit dem Schriftzug „Rabini Schem Ha Mphoras“ versehen ist. Das Relief stammt ursprünglich etwa aus dem 13. Jahrhundert; der Schriftzug wurde um das Jahr 1570 angebracht. Das Relief stellt ein Schwein („Judensau“) dar, an dessen Zitzen Menschenkinder säugen, die durch ihre Spitzhüte als Juden identifiziert werden sollen. Eine ebenfalls durch einen Hut als Rabbiner zu identifizierende Figur hebt mit der Hand den Schwanz der Sau und blickt ihr in den After (vgl. Fotografien Anlagen B 3, Bd. I BI. 39 d.A., B 9). Der Kläger macht einen Anspruch auf Beseitigung des Sandsteinreliefs geltend.
Am Fuße der Stadtkirche, unter dem Relief, befinden sich ein als Bodenreliefplatte ausgeführtes Mahnmal, ein mit einem Informationstext versehener Schrägaufsteller (vgl. Fotografie Anlage B 7, Bd. I Bl. 114 d.A.) sowie eine - als Symbol Israels ausgewählte - Zeder, die von der Beklagten als Zeichen der Versöhnung zwischen Juden und Christen verstanden wird.

Der Kläger hat die Auffassung vertreten, durch ihr Festhalten an der Ausstellung der "Judensau“ begehe die Beklagte eine Beleidigung im Sinne von $\S 185$ StGB; sie nehme die beleidigende Wirkung der Schmähskulptur zumindest billigend in Kauf, selbst wenn sie sich die beleidigende Wirkung der Schmähskulptur nicht zu Eigen machen wolle. Zugleich liege ein Verstoß gegen das allgemeine Persönlichkeitsrecht vor.

Die Beklagte hat gemeint, weder den Kläger noch andere Menschen jüdischen Glaubens zu beleidigen. Auch ein Eingriff in das allgemeine Persönlichkeitsrecht des Klägers liege nicht vor. Denn die Plastik sei in ein Ensemble von Gedenkelementen eingebettet, welches die bedrückende Geschichte erkläre und die moralische Verantwortung der heute in ... I e b e $n$ d e $n$ Christen für das von ihren Vorfahren den Juden zugefügte Leid anerkenne.

Hinsichtlich der Einzelheiten des erstinstanzlichen Sach- und Streitstandes einschließlich der gestellten Anträge wird auf die tatsächlichen Feststellungen der angefochtenen Entscheidung (Bd. II Bl. 3 ff. d.A.) Bezug genommen.

Das Landgericht hat die Klage abgewiesen. Ein Beseitigungsanspruch analog § 1004 BGB bestehe nicht, da die bildliche Darstellung in einem objektiven Sinn nicht als Kundgabe der eigenen Missachtung im Sinne von $\S 185$ StGB verstanden werden könne; das Relief sei Bestandteil einer "Gedenkkultur". Hinsichtlich der Einzelheiten wird auf die Entscheidungsgründe des angefochtenen Urteils (Bd. II Bl. 7 ff. d.A.) Bezug genommen.

Gegen dieses Urteil wendet sich die Berufung des Klägers. Der Kläger beanstandet, dass das Landgericht bei seiner - zutreffenden - Einschätzung, dass nach dem Schwerpunkt des Sachverhalts ein aktives Tun seitens der Beklagten vorliege und kein schlichtes Unterlassen, die Wiederherstellungsarbeiten der Beklagten hinsichtlich der Steinplastik Ende der 80er Jahre nicht als relevant gewürdigt worden seien. Von einer ordnungsgemäßen Subsumtion der erforderlichen Tatbestandsmerkmale auf den vorliegenden Fall könne nicht gesprochen werden. Es werde nicht nachvollziehbar dargelegt, warum das jeweilige 
Kommentarzitat auf den Sachverhalt zutreffe, und ob die vom Gericht gezogenen Konsequenzen aus der Sachverhaltsauslegung tatsächlich den zitierten Kommentarstellen entsprächen.

Entgegen den Ausführungen des Landgerichts erfülle das Ausstellen der Schmähplastik nicht nur den Tatbestand einer Beleidigung, sondern auch den Tatbestand einer Formalbeleidigung als Sonderfall einer Beleidigung. Entgegen den Ausführungen des Urteils komme es für die Einschätzung, ob eine Formalbeleidigung vorliege, nicht allein auf den "objektiven Sinngehalt einer Äußerung unter Beachtung der Begleitumstände und des Gesamtzusammenhanges sowie der zeitlichen und örtlichen Umstände" an. Das Gericht hätte sich vertiefter mit der "objektiven Wahrnehmung" der "Judensau" unbeteiligter Dritter auseinandersetzen müssen. Eine Beleidigung bleibe eine Beleidigung, ob man sie kommentiere oder nicht, wie der jetzige Landesbischof der Evangelischen Kirche in Mitteldeutschland - unstreitig - formuliert habe.

Die Frage, ob die von der Beklagten herangezogene Gedenkkulturargumentation etwas an dem objektiven Sinngehalt der Schmähskulptur ändern könne, werde im angegriffenen Urteil an der falschen Stelle behandelt. Die Tatbestandlichkeit einer Formalbeleidigung sei zwischen den Parteien nicht im Streit, so dass es allenfalls um einen Rechtfertigungs- oder Entschuldigungsgrund gehe.

Entgegen der erstinstanzlichen Entscheidung habe die Beklagte mit bedingtem Vorsatz gehandelt, da sie von der für jüdische Menschen beleidigenden Wirkung der Skulptur gewusst habe.

Die Verfolgung eines möglicherweise berechtigten Zwecks, der in einer Verfolgung einer angemessenen Gedenkkultur liege, rechtfertige die Ehrverletzung allein noch nicht. Zudem sei das Mahnmal der Beklagten unterhalb der Schmähskulptur völlig ungeeignet, um die beleidigende Wirkung der „Judensau“ $z u$ verringern. Es nehme zudem wie die gepflanzte Zeder nicht auf die "Judensau" Bezug. Die "Judensau" müsse vielmehr in eine museale Aufarbeitung in einem geschlossenen, nicht öffentlichen Raum eingebunden werden; sie dürfe nicht unbegrenzt und unbeeinflussbar in den öffentlichen Raum hineinwirken.

Die Erfüllung des Tatbestands einer rechtswidrigen Beleidigung indiziere auch die schuldhafte Begehung.

Der Beseitigungsanspruch sei auch wegen Verletzung des allgemeinen Persönlichkeitsrechts begründet.

Der Kläger beantragt, unter Abänderung des Urteils des Landgerichts Dessau vom 24.05.2019, Az. 2 O 230/18, die Beklagte und Berufungsbeklagte zu verurteilen, das Sandsteinrelief „Judensau“ vom Südostflügel der Stadtkirche ... zu entfernen bzw. entfernen zu lassen, und vorsorglich, im Übrigen feststellen zu lassen, dass das am Südostflügel der Stadtkirche ... auf etwa vier Meter Höhe angebrachte Sandsteinrelief, bezeichnet als „Judensau“, den objektiven und subjektiven Tatbestand der Beleidigung gemäß § 185 StGB erfüllt.

Die Beklagte beantragt, die Berufung zurückzuweisen.

Sie verteidigt das angegriffene Urteil.

Hinsichtlich der weiteren Einzelheiten des Sach- und Streitstandes wird auf die Schriftsätze der Parteien nebst Anlagen und auf die Protokolle der mündlichen Verhandlungen Bezug genommen.

\section{II.}

Die Berufung ist zulässig; sie bleibt aber in der Sache ohne Erfolg.

1. Die vom Landgericht problematisierte Frage der Eröffnung des Rechtswegs zu den ordentlichen Gerichten ist gemäß § 17a Abs. 5 GVG im Berufungsverfahren nicht zu prüfen. Keine Partei hat in erster Instanz die Zulässigkeit des Rechtswegs gerügt, so dass eine Vorabentscheidung über die Zulässigkeit des beschrittenen Rechtswegs nicht gemäß § 17a Abs. 3 GVG geboten war; das Landgericht hat bei seiner Entscheidung in der Hauptsache seine Zuständigkeit bejaht.

2. Der Kläger hat gegen die Beklagte keinen Anspruch auf Beseitigung des an der Stadtkirche von ... befindlichen "Judensau"-Reliefs in analoger Anwendung von § 1004 BGB in Verbindung mit $\S \S 823$ Abs. 2, 31 BGB, 185 StGB oder wegen Verletzung des aus Art. 1, 2 GG abgeleiteten allgemeinen Persönlichkeitsrechts.

a) Unstreitig befindet sich an der Stadtkirche von ...entsprechend dem Vorbringen des Klägers das von diesem beanstandeten Relief. Die Parteien stimmen auch dahingehend überein, dass dieses Relief zur Zeit seiner Entstehung und auch noch im 16. Jahrhundert, als es durch die Inschrift "Schem Ha Mphoras" ergänzt wurde, dazu diente, Juden verächtlich zu machen.

b) Der Kläger kommt - was von der Beklagten nicht angezweifelt wird - als Jude als möglicher Anspruchsinhaber in Betracht, auch wenn nicht er persönlich in dem streitgegenständlichen Relief dargestellt ist. Ungeachtet ihrer Zahl wird von der Rechtsprechung die Kollektivbeleidigungsfähigkeit der in Deutschland lebenden Juden bejaht, was damit zu begründen ist, dass sie wegen des in der Geschichte einmaligen, ihnen vom Nationalsozialismus auferlegten Schicksals in der Allgemeinheit als eine eng umgrenzte Gruppe erscheinen (Eisele/ Schittenhelm, in: Schönke/Schröder, StGB, 30. Auflage, vor §§ 185 ff. Rn. 7b und 8d).

c) Die Beklagte ist passivlegitimiert. Auch wenn nicht sie selbst den Tatbestand des § 185 StGB verwirklichen kann, sondern allenfalls das für sie handelnde Organ, der Vorsitzende des 
Gemeindekirchenrates, haftet die Beklagte, soweit ein Beseitigungsanspruch wegen analoger Anwendung von § 823 Abs. 2 BGB iVm § 185 StGB in Betracht kommt, zivilrechtlich über die Zurechnungsnorm des $\S 31$ BGB. Soweit es um einen Beseitigungsanspruch wegen Verletzung des allgemeinen Persönlichkeitsrechts des Klägers geht, haftet die Beklagte unmittelbar.

d) Tathandlung einer Beleidigung ist die Kundgabe der Missachtung oder Nichtachtung, d.h. deren Manifestation durch ein Verhalten mit einem entsprechenden Erklärungswert, gleichgültig, ob es sich dabei um Äußerungen durch Wort, Schrift, Bild, Gesten, symbolische Handlungen oder Tätlichkeiten handelt. Maßgebend dafür, ob eine Äußerung die Missachtung eines anderen zum Ausdruck bringt, ist nicht, wie der Täter sie versteht oder wie der Empfänger sie tatsächlich verstanden hat, sondern wie dieser sie verstehen durfte, d.h. ihr durch Auslegung zu ermittelndem objektivem Sinngehalt. Die Gesamtheit der äußeren und inneren Umstände ist zu berücksichtigen, insbesondere der Ton, Alter, Stellung, persönliche Eigenschaften und Beziehungen der Beteiligten, die Anschauungsweise der beteiligten Kreise und ihre Gewöhnung an bestimmte Redewendungen, die Ortsüblichkeit bestimmter Ausdrücke, die Umstände, unter denen die Äußerung erfolgte usw. Eine Äußerung darf nicht aus dem sie betreffenden Kontext herausgelöst einer rein isolierten Betrachtung zugeführt werden (Eisele/Schittenhelm, StGB, 30. Auflage, § 185 Rn. 8; entsprechend Regge/Pegel, in Münchener Kommentar zum StGB, 3. Auflage, § 185 Rn. 8 und 10; BGH, Urteil vom 18.02.1964, 1 StR 572/63; Urteil vom 12.04.2016, VI ZR 505/14, Rn. 11).

e) Das Verhalten der Beklagten, das an den genannten Maßstäben zu messen ist, besteht darin, dass sie das Relief an der Kirchenmauer für die Öffentlichkeit sichtbar vorhält. Die Beklagte ist für den jetzigen Zustand der Kirche, insbesondere für das Vorhandensein des Reliefs, verantwortlich nach Maßgabe der derzeit gültigen Gesetze. Die Beklagte hat sich bewusst spätestens seit den 80 er Jahren des 20. Jahrhunderts und bis in die heutige Zeit für die Beibehaltung des Reliefs entschieden; darin, dass sie das Relief für die Öffentlichkeit sichtbar vorhält, liegt ein positives Tun, nicht lediglich ein Unterlassen. Ob sie es war, die die Herstellung des Reliefs im 13. Jahrhundert und seine Anbringung an die Kirchenwand veranlasst hat, ist nicht entscheidend. Auch der Umfang der Restaurierungsarbeiten in den 80er Jahren des 20. Jahrhunderts ist in diesem Zusammenhang unerheblich.

Das Zurschaustellen des Reliefs wäre bei isolierter Betrachtung nach heutigen Wertvorstellungen als Beleidigung von Juden zu werten. Die Beklagte selbst bezeichnet das Relief als Schmähplastik. Die an dem Relief angebrachte, an eine antijudaistische Schrift Martin Luthers angelehnte Inschrift verstärkt deren beleidigende Wirkung. Die Beeinträchtigung der Ehre bzw. des Persönlichkeitsrechts eines Juden durch die aus der bildlichen Darstellung ersichtliche, bei Anbringung des Reliefs an die Kirchenwand ursprünglich angestrebte Verhöhnung und Herabwürdigung der Juden stände gegenüber etwaigen schüt- zenswerten Interessen der Beklagten, die dieser aufgrund ihres Eigentumsrechts an dem Kirchengebäude, ihrer allgemeinen Handlungsfreiheit, der Freiheit der Religionsausübung oder aufgrund der gemäß Art. 140 GG zum Gegenstand des Grundgesetzes gewordenen Artikel 136-139 und 141 der Weimarer Reichsverfassung zustehen, vollständig im Vordergrund. Dass das Relief einen nennenswerten künstlerischen Rang habe, wird von der Beklagten nicht behauptet; die Frage der Abwägung zwischen Persönlichkeitsrecht und Kunstfreiheit stellt sich mithin im vorliegenden Fall nicht.

Bei Auslegung aller Umstände ergibt sich allerdings im vorliegenden Fall unter Berücksichtigung der konkreten Umstände des Sachverhalts als objektiver Sinngehalt, dass die Beklagte mit der Zurschaustellung des Reliefs an der Kirchenwand nicht die Ehre der Juden und somit auch nicht die Ehre des Klägers verletzt, weil das Relief inzwischen Teil eines Ensembles von Exponaten ist, das eine andere Zielrichtung der Beklagten erkennen lässt, nämlich diejenige, das Relief in Verbindung mit dem auf einem Schrägaufsteller lesbaren Informationstext und einem Mahnmal als Teil einer Gedenk- und Erinnerungskultur zu erhalten. Bereits der objektive Tatbestand des $\S 185$ StGB ist nicht verwirklicht; auch ein Verstoß gegen das Persönlichkeitsrecht des Klägers liegt nicht vor.

Wer das Relief betrachtet, kann nach den örtlichen Verhältnissen, wie sie sich auf den zur Akte gereichten Fotos darstellen, die in unmittelbarer Nähe aufgestellte Informationstafel und das Mahnmal nicht übersehen. In dem Informationstext auf dem Schrägaufsteller wird auf die Plastik hingewiesen, die sich seit etwa 1290 an der Südostecke der Stadtkirche ...befinde. Die Plastik wird als Hohn- und Spottbild auf die jüdische Religion bezeichnet. Weiter wird ausgeführt, dass Schmähplastiken dieser Art, die Juden in Verbindung mit Schweinen zeigten - Tiere, die im Judentum als unrein gelten - besonders im Mittelalter verbreitet gewesen seien. Weiter wird auf Judenverfolgungen in Sachsen Anfang des 14. Jahrhunderts und 1440 hingewiesen, sowie darauf, dass 1536 Juden der Aufenthalt in Sachsen grundsätzlich verboten worden sei. Weiter wird in dem Informationstext ausgeführt, dass Martin Luther 1543 die antijudaistischen Schriften „Von den Juden und ihren Lügen“ sowie „Vom Schem Hamphoras und vom Geschlecht Christi" veröffentlicht habe, auf die sich die Inschrift der Schmähplastik beziehe, die 1570 angebracht worden sei. Dieser Informationstext bringt, soweit es bei einem auf Mitteilung objektiver Fakten abzielenden Text möglich ist, unmissverständlich zum Ausdruck, dass die Beklagte sich von den Judenverfolgungen, den antijudaistischen Schriften Luthers und insbesondere auch von der verhöhnenden und verspottenden Zielrichtung der Schmähplastik distanziert. Dies wird bekräftigt dadurch, dass der Informationstext auch auf das im Jahr 1988 enthüllte, an den Tod von sechs Millionen Juden unter einem Kreuzeszeichen erinnernde Mahnmal unterhalb der Schmähplastik verweist. Das Mahnmal selbst nimmt zwar nicht direkt auf die in dem Relief enthaltene bildliche Darstellung Bezug, mit der Formulierung „... der geschmähte Schem Ha Mphoras ..." wohl aber - und zwar in distanzierender Weise 
- auf die an dem Relief angebrachte Inschrift. Für den Leser des Informationstextes wird damit die Verbindung der Schmähplastik, des Mahnmals und des Schrägaufstellers mit dem Informationstext zu einem Ensemble von Objekten des Gedenkens und der Erinnerung verdeutlicht. Die Beklagte zitiert praktisch die Aussage des Reliefs in direkter Rede, eingebettet in die Darstellung der historischen Einordnung. Mit dem Ensemble von Exponaten soll an die jahrhundertelange Diskriminierung und Verfolgung von Juden, auch unter maßgeblicher Mitwirkung der Kirche, und - durch das Mahnmal - an die Shoa erinnert werden. Bei objektiver Betrachtung stellt das Ensemble von Exponaten einschließlich der Schmähplastik keine Kundgabe der Missachtung oder Nichtachtung von Juden dar. Dies ist auch für Juden, die das Relief, den Schrägaufsteller mit dem Informationstext und das Mahnmal betrachten, erkennbar; ausweislich seiner Ausführungen auf S. 4 f. der Klageschrift hat auch der Kläger zur Kenntnis genommen, dass die Beklagte sich die Beleidigungswirkung der Schmähskulptur nicht zu Eigen machen will. Ob der Kläger sich durch das Vorhandensein des Reliefs am ursprünglichen Ort subjektiv dennoch in seiner Ehre bzw. in seinem Persönlichkeitsrecht verletzt fühlt, und ob die Beklagte derartige Gefühle von Juden für möglich hält, ist nach den gefestigten Rechtsprechungsgrundsätzen, an denen der Senat festhält, nicht entscheidend, weil dafür, ob der objektive Tatbestand einer Beleidigung erfüllt ist, der erkennbare objektive Sinngehalt der Verlautbarung maßgeblich ist.

f) Zugespitzt läuft die Argumentation des Klägers auf den sinngemäß vom jetzigen Bischof der Evangelischen Kirche in Mitteldeutschland formulierten Gedanken hinaus, dass eine Beleidigung auch dann eine Beleidigung bleibe, wenn man sie kommentiere. Konsequenterweise müsste mit dieser Erwägung allerdings auch eine Ausstellung des Reliefs in einem Museum, wie sie der Kläger befürwortet, abgelehnt werden. Auch der Träger des betreffenden Museums würde gegebenenfalls das Relief, das bei isolierter Betrachtung eine Beleidigung darstellt, öffentlich zur Schau stellen, ergänzt um eine Kommentierung durch erläuternde Texte. Auch wenn die Zahl der Besucher eines Museums in der Regel niedriger ist als die Zahl der Passanten, die an einem Kirchengebäude vorbeigehen, ist ein Museum - wenn auch gegen Eintritt - öffentlich zugänglich. Nach der These, dass eine Beleidigung auch dann eine Beleidigung sei, wenn man sie kommentiere, müsste der Träger des Museums ebenfalls eine Beleidigung bzw. eine Persönlichkeitsrechtsverletzung begehen. Wenn generell der beleidigende Aussagegehalt eines Exponats durch Kommentierungen nicht neutralisiert werden könnte, wäre dies in einem Museum im gleichen Maße der Fall, wie wenn sich das zur Schau gestellte Objekt an der Außenwand eines Kirchengebäudes befindet. Dies zeigt, dass der Gedanke, eine Beleidigung bleibe auch dann eine Beleidigung, wenn man sie kommentiere, nicht generell und ausnahmslos gelten kann.

Eine Ausstellung des streitgegenständlichen Reliefs in einem Museum würde zudem nicht zwangsläufig die Gewähr dafür bieten, dass die Erläuterung des Reliefs die Zielrichtung hätte, kritisch und distanziert über den bereits vor Jahrhunderten verbreiteten Antisemitismus in Deutschland zu informieren. Hier käme es auf den Zweck an, den das Museum mit der Präsentation verfolgt; eine Ausstellung in einem Museum kann theoretisch auch darauf abzielen, den Gegenstand der musealen Darstellung bei dem Betrachter im günstigen Licht erscheinen zu lassen. Dies ist unabhängig davon, wie viele Personen ein Exponat sehen, ob lediglich ein begrenzter Personenkreis, der für den Eintritt in das Museum bezahlt, oder zahlreiche Passanten, die an der Stadtkirche vorbeigehen. Der Betrachter sieht das Objekt sowohl im Museum wie an der Außenwand des Kirchengebäudes gegebenenfalls im Zusammenhang mit Informationstafeln, die sich auf das Objekt beziehen.

Der Unterschied zwischen einer Kirche wie hier der Stadtkirche ... und einem reinen Museum besteht im Wesentlichen darin, dass die Stadtkirche in erheblichem Umfang zu kirchlichen Zwecken, insbesondere zur Abhaltung von Gottesdiensten, genutzt wird. Bei Fehlen von vor Ort befindlichen Informationstafeln zur historischen Einordnung bestände durchaus die vom Kläger gesehene Gefahr, dass die Schmähplastik als Teil der religionsimmanenten Verkündigung wahrgenommen werden müsste. Indes ist die Bildung des durch den Inhalt des Informationstextes und durch räumliche Nähe der Bestandteile gekennzeichneten Ensembles aus Mahnmal, Informationstafel und Relief als Begründung einer Teilfunktion des Kirchengebäudes als Museum und Gedenkstätte zu verstehen; durch diese Einbindung in das Ensemble von Objekten des Gedenkens und der Erinnerung wird der Gefahr, dass das Relief mit seiner ursprünglichen, herabwürdigenden Zielsetzung Bestandteil der Verkündigung wird, wie der Kläger meint, entgegengewirkt.

Soweit der Kläger in der mündlichen Verhandlung vor dem Senat einen Unterschied zwischen der Präsentation am Originalstandort und in einem Museum darin gesehen hat, dass in einem Museum anders als außerhalb einer Kirche Hausrecht ausgeübt werden könne, und dass bei einem Verbleib des Reliefs an der Kirchenaußenwand die Gefahr der Nachahmung durch Neo-Nationalsozialisten bestehe, ist dies ein Gesichtspunkt, der für die Gefahrenabwehr von Belang sein kann, insoweit auch für die Beklagte als Grundstückseigentümerin und mögliche Zustandsstörerin, nicht aber für die Wertung, ob das Zurschaustellen der Schmähplastik eine Beleidigung bzw. Persönlichkeitsrechtsverletzung darstellt oder nicht. Der Schutzzweck des $§ 185$ StGB und auch des allgemeinen Persönlichkeitsrechts geht nicht dahin, Ausschreitungen in der Umgebung der Stadtkirche oder eine positive Bewertung des Reliefs durch Neo-Nationalsozialisten zu verhindern.

Ob der Inhalt des im Bereich des Mahnmals und des Reliefs aufgestellten Informationstextes maximalen Qualitätsansprüchen genügt, kann hier dahingestellt bleiben. Wie viele und welche Informationen bei musealer Präsentation - an welchem Ort auch immer - sinnvollerweise gegeben werden sollten, ist wie die Frage, ob das Relief am ursprünglichen Standort verbleiben, in ein unter freiem Himmel aufgestell- 
tes gesondertes Mahnmal integriert oder in einem geschlossenen Museumsraum ausgestellt wird, und wie die Frage, in welcher Weise gegebenenfalls Scham oder Buße zu erkennen gibt, im gesellschaftlichen Rahmen zu diskutieren, auch unter Berücksichtigung öffentlich-rechtlicher Gesichtspunkte, etwa des Denkmalschutzes. Für die rechtliche Wertung, dass die Zurschaustellung des Reliefs im vorliegenden Fall keine Beleidigung oder Verletzung des Persönlichkeitsrechts des Klägers darstellt, ist entscheidend, dass im vorliegenden Fall durch den Informationstext die Distanzierung der Beklagten von dem schmähenden Charakter der Plastik deutlich gemacht wird wie auch das von der Beklagten verfolgte Ziel, die Plastik lediglich im Rahmen einer Kultur des Gedenkens an jahrhundertelange Judenverfolgung zu erhalten.

g) Die Parteien haben in erster Instanz Erwägungen dazu angestellt, inwieweit die an Konzentrationslagern angebrachten Mottosprüche "Arbeit macht frei“ oder "Jedem das Seine" mit der an der Stadtkirche von ...angebrachten "Judensau"-Darstellung vergleichbar ist. Die Mottosprüche sind aus heutiger Perspektive nur deswegen nicht mehr als Beleidigung von Juden zu verstehen, weil der Betreiber der jeweiligen Gedenkstätte den gesamten Gebäudekomplex durch die Art der Präsentation in der Weise umgewidmet hat, dass der Gebäudekomplex einschließlich der Texte als Mahnmal zur Erinnerung an die Vergasung von Millionen Juden dargestellt wird, wodurch Juden eben gerade nicht beleidigt werden. Die Mottosprüche in den Konzentrationslagern haben den ursprünglichen Charakter der Beleidigung nur durch die Art der Präsentation seitens der Träger der jeweiligen Gedenkstätte verloren. Hier kann durchaus eine Parallele zu der hier streitgegenständlichen Situation an der Außenwand der Stadtkirche von ... gesehen werden; sie bestätigt, dass bei einer über die historische Einordnung kommentierenden, den originalen Bauzustand erhaltenden Präsentation eines ursprünglich beleidigend gemeinten Teils einer baulichen Anlage der beleidigende Charakter neutralisiert werden kann, wie es bei der streitgegenständlichen Schmähplastik der Fall ist.

Einen Vergleich stellt der Kläger sowohl in erster Instanz als auch im Schriftsatz vom 08.01.2020 auch zu dem Fall des "Schmähgedichts" des Satirikers Böhmermann über den türkischen Staatspräsidenten Erdogan an (vgl. OLG Hamburg, Urteil vom 15.05.2018, 7 U 34/18). In jenem Fall hat das Oberlandesgericht Hamburg auf die Würdigung der Gesamtumstände abgestellt, aus denen sich ergab, dass trotz der Kommentierung, derzufolge eine Beleidigung nicht beabsichtigt sei, dennoch eine Beleidigung vorlag, weil dies aus der Art des Vortrags geschlossen werden konnte. Aus dieser Entscheidung ist entgegen der Meinung des Klägers nicht zu folgern, dass in jedem Fall ein zu einer Beleidigung abgegebener Kommentar des Inhalts, dass eine Beleidigung nicht vorliegen soll, unerheblich sein soll. Im dortigen Fall machen dem OLG Hamburg zufolge der Ton und die Art des Vortrags, in dem um das Gelächter des Publikums geradezu gebuhlt wird, vor allem aber der Umstand, dass der Vortrag der Verse letztlich doch auch der Kritik an einem Verhalten des dortigen Klägers dienen solle, den Zuschauern deutlich, dass der vorangestellte Vorbehalt, nicht beleidigen zu wollen, tatsächlich nicht greife, weil es eben doch darum gehe, den dortigen Kläger in schwerer Weise zu beleidigen. Hier ist der Sachverhalt anders gelagert, weil die Art der Präsentation des Reliefs durch die Zuordnung einer in der Nähe befindlichen Informationstafel und eines Mahnmals erkennbar darauf abzielt, dass die Beklagte Juden nicht beleidigen will.

h) Nicht entscheidend sind für die rechtliche Wertung die von der Beklagten angeführten weiteren Bestandteile der Gedenkkultur, weil diese nicht in engem räumlichen Zusammenhang mit dem Sandsteinrelief stehen, in dem auf dem Schrägaufsteller angebrachten Informationstext nicht erwähnt werden und deshalb vom Betrachter nicht als zu demselben Ensemble gehörig wahrgenommen werden müssen. Das gilt zunächst für die Stadtführungen. Diese werden nicht von der Beklagten verantwortet; nicht jeder Passant nimmt zudem an einer Stadtführung teil. Auch der Internetauftritt, der Informationen und Stellungnahmen der Beklagten zu der Problematik der Schmähplastik bietet, ist als solcher unabhängig von dem Eindruck, den das Sandsteinrelief im Zusammenhang mit der Informationstafel und dem Mahnmal vermittelt; wer diese Gedenkstätte betrachtet, liest typischerweise nicht gleichzeitig die Ausführungen im Internetauftritt der Beklagten. Entsprechendes gilt für Informationsblätter, die die Beklagte anbietet; derartige Blätter werden vom Betrachter möglicherweise, aber nicht zwangsläufig bei der Betrachtung der Plastik gelesen. Auch die in der Nähe des aus Schmährelief, Schrägaufsteller und Mahnmal befindliche Zeder bleibt bei der rechtlichen Bewertung des Reliefs außer Betracht. Die Beklagte versteht die Zeder zwar als Zeichen der Versöhnung; es ist aber nicht dargelegt, aufgrund welcher Anhaltspunkte der Passant, der die Informationstafel auf dem Schrägaufsteller und insbesondere das Schmährelief sieht, dieses mit der Pflanzung der Zeder verfolgte Ziel erschließen soll. Auch Gedenkveranstaltungen, die zu bestimmten Anlässen im Bereich des Mahnmals stattfinden, beeinflussen die Bewertung des Reliefs nicht, da diese Veranstaltungen von den meisten Passanten und Besuchern nicht wahrgenommen werden.

i) Soweit der Kläger mit dem Begriff der Formalbeleidigung argumentiert, verhilft inm dies nicht zum Erfolg. Der Terminus der Formalbeleidigung wird üblicherweise, wie das angegriffene Urteil ausführt, bei Anwendung von § 192 StGB als Kundgabe eines an sich zutreffenden Sachverhalts verstanden, der lediglich durch die gewählte Form oder durch sonstige besondere äußere Umstände, in denen der Sachverhalt verbreitet wird, als Ehrverletzung verstanden wird (vgl. Regge/Pegel, in Münchener Kommentar zum StGB § 192 Rn. 1; Eisele/Schittenhelm in Schönke/Schröder, 30. Auflage $\S 192 \mathrm{Rn}$. 1). Das beanstandete Relief stellt als solches unzweifelhaft keine Tatsachenbehauptung dar, weder eine zutreffende noch eine unzutreffende; und die Erläuterung durch den Schrägaufsteller begründet ersichtlich nicht den entehrenden Charakter des Reliefs. 
j) Entgegen der in erster Instanz vertretenen Meinung des Klägers kommen §§ 19, 21 AGG nicht als mögliche Anspruchsgrundlage in Betracht; der hier vorliegende Sachverhalt ist nicht vom Anwendungsbereich des AGG, der in § 2 AGG normiert ist, erfasst. Dort geht es anders als im vorliegenden Fall um die Bedingungen der Erwerbstätigkeit, Zugang zur Berufsberatung, Mitgliedschaft in einer Beschäftigten- oder Arbeitgebervereinigung, Sozialschutz, Bildung und den Zugang zu Gütern und Dienstleistungen.

3.

Der hilfsweise gestellte Feststellungsantrag ist für den Fall gestellt worden, dass nach Meinung des Senats eine Verurteilung der Beklagten auf den Beseitigungsantrag daran scheitert, dass eine Beseitigung der Skulptur aus Denkmalschutzgründen unmöglich ist. Der Hilfsantrag ist nicht zur Entscheidung angefallen; die Prozessbedingung ist nicht eingetreten; der Beseitigungsantrag wird unabhängig von einer aus öffentlichrechtlichen Gründen bestehenden Unmöglichkeit der Beseitigung abgewiesen.

\section{III.}

Die Entscheidung über die Kosten des Berufungsverfahrens beruht auf $\S 97$ Abs. 1 ZPO, die Entscheidung über die vorläufige Vollstreckbarkeit auf $\S \S$ 708 Nr. 10, 709 S. 2, 711 ZPO.

Der Senat hat gemäß § 543 Abs. 2 Nr. 1 ZPO die Revision zugelassen; die Rechtssache hat grundsätzliche Bedeutung. In Deutschland befinden sich an zahlreichen Kirchengebäuden Darstellungen, die zur Zeit ihrer Entstehung der Verhöhnung von Juden dienten. Darüber hinaus stellt sich die hier maßgebliche Frage, wie mit Herabwürdigungen von Personengruppen, die in älteren bildlichen oder textlichen Darstellungen zum Ausdruck kommen enthalten, heutzutage in zivilrechtlicher Hinsicht umzugehen ist, auch in anderen Zusammenhängen.

Die Streitwertfestsetzung beruht auf $\S \S 43,47$, 48 Abs. 1, 63 Abs. 2 GKG, 3 ZPO.

Für die Streitwertfestsetzung sind nicht, wie der Kläger ausweislich der Klageschrift meint, die mutmaßlichen Beseitigungskosten maßgeblich; der Kläger macht wegen Persönlichkeitsrechtsverletzung bzw. Ehrverletzung einen nichtvermögensrechtlichen Anspruch geltend, dessen Wert gemäß § 48 Abs. 2 GKG festzusetzen ist (vgl. Schneider/Volpert/ Fölsch, Gesamtes Kostenrecht, 2. Auflage, § 48 GKG
Rn. 15). Der vom Kläger in der Klageschrift angegebene Streitwert 10.000,00 $€$ ist aber auch unter dem Gesichtspunkt einer Persönlichkeitsrechtsverletzung oder Ehrverletzung bei Berücksichtigung einerseits der Bedeutung der Sache und andererseits des in tatsächlicher Hinsicht begrenzten Umfangs der Streitigkeit ein hinreichend tragfähiges Indiz, das eine Festsetzung des Streitwerts auf $10.000,00 €$ rechtfertigt.

Buchloh, Tauscher, Dr. Strietzel 